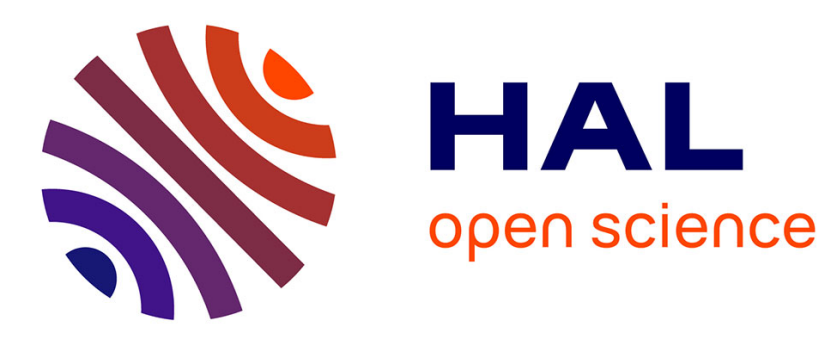

\title{
Polyhedral Bubble Vibrations
}

Mariem Boughzala, Olivier Stephan, Emmanuel Bossy, Benjamin Dollet, Philippe Marmottant

\section{To cite this version:}

Mariem Boughzala, Olivier Stephan, Emmanuel Bossy, Benjamin Dollet, Philippe Marmottant. Polyhedral Bubble Vibrations. Physical Review Letters, 2021, 126 (5), pp.054502. 10.1103/PhysRevLett.126.054502 . hal-03382369

\section{HAL Id: hal-03382369 https://hal.science/hal-03382369}

Submitted on 18 Oct 2021

HAL is a multi-disciplinary open access archive for the deposit and dissemination of scientific research documents, whether they are published or not. The documents may come from teaching and research institutions in France or abroad, or from public or private research centers.
L'archive ouverte pluridisciplinaire HAL, est destinée au dépôt et à la diffusion de documents scientifiques de niveau recherche, publiés ou non, émanant des établissements d'enseignement et de recherche français ou étrangers, des laboratoires publics ou privés. 


\title{
Polyhedral Bubble Vibrations
}

\author{
Mariem Boughzala, Olivier Stephan, Emmanuel Bossy, Benjamin Dollet $\odot$, and Philippe Marmottant $\odot^{*}$ \\ University Grenoble Alpes, CNRS, LIPhy, F-38000 Grenoble, France
}

(Received 23 November 2020; accepted 13 January 2021; published 5 February 2021)

\begin{abstract}
Underwater bubbles are extremely good acoustic resonators, but are freely evolving and dissolving. Recently it was found that bubbles can be stabilized in frames, but the influence of the frame shape is still undocumented. Here we first explore the vibration of polyhedral bubbles with a low number of faces, shaped as the five Platonic solids. Their resonance frequency is well approximated by the formula for spherical bubbles with the same volume. Then we extend these results to shapes with a larger number of faces using fullerenes, paving the way to obtain arbitrary large resonant bubbles.
\end{abstract}

DOI: 10.1103/PhysRevLett.126.054502

Introduction.-Bubbles in water react vividly under acoustic waves because of the compressibility of their constitutive gas, featuring ample vibrations compared to liquid or solid surroundings. Bubbles are good acoustic vibrators with a marked resonance frequency [1,2], useful to absorb or reemit vibrations in materials containing many of them [3-7]. Bubbles are strongly influenced by each other even at a distance, responding to the vibration waves emitted by neighbor bubbles. A lot of vibration without contact is reminiscent of platonic love in human interactions. Here, we will consider the vibration of a lone bubble of Platonic shape.

Free bubbles in water, spherical in shape, are unstable in position (they float) or in volume (their gas dissolves in water [8,9]). In a previous work we introduced cubic bubbles: air pockets trapped in 3D-fabricated cubic frames immersed under water. The frame stabilizes both the bubble position and volume for more than a day [10] and enables us to build an array of such bubbles [11] useful to create new acoustic metamaterials. Capillary forces prevent water from entering the frames if the openings are small enough (a maximum aperture of $2.1 \mathrm{~mm}$ was reported for a cube, a dimension comparable to the capillary length $l_{c}=\sqrt{\sigma / \rho_{l} g}$, with $\sigma$ the surface tension, $\rho_{l}$ the liquid density, and $g$ the gravitational constant). The frame does not impede vibrations of the liquid-gas interfaces, that are flat at equilibrium.

The cubic shape is only one specific shape, chosen because of its ease in 3D design. Our scientific question is now to understand the vibration of other shapes, and to determine what is the maximal bubble size of these shapes.

In this Letter we explore arbitrary shapes starting with regular polyhedra: the five Platonic solids, namely tetrahedron, cube, octahedron, dodecahedron, and icosahedron. We thus introduce platonic bubbles, featuring, respectively, $4,6,8,12$, and 20 faces. Eventually we extend our study to bubbles with an arbitrary number of faces.

Millimetric frames representing the five solids were manufactured by $3 \mathrm{D}$ stereolithography in photoresist that is rendered hydrophobic by silanization to disadvantage the entry of water (see further details in Supplemental Material [12]). Frames are slowly immersed under water, keeping bubbles trapped and creating roughly flat interfaces at the openings (Fig. 1).

Acoustic vibrations of Platonic bubbles.-Platonic frames are slowly immersed in a water tank and the acoustic vibration of the formed enclosed bubble is excited in the near field of an underwater loudspeaker [Fig. 2(a)], sweeping a large range of frequencies. The acoustic pressure $P(t)$ is recorded with a hydrophone placed as close as possible to the bubble to increase the signal amplitude. A reference pressure signal $P^{0}(t)$ is measured at the same position without the bubble, in order to isolate the contribution of the bubble. The relative contribution of the bubble to the spectrum of the signal is estimated by $A=$ $\left(\hat{P}-\hat{P}^{0}\right) / \hat{P}^{0}$, where $\hat{P}$ and $\hat{P}^{0}$ are the Fourier transforms.

The bubble resonance is clearly evidenced by looking at the amplitude of the bubble contribution $A$ which goes through a maximum [norm of $A$ plotted on Fig. 2(b)]. A more precise measurement of the resonance frequency is found by looking at the cosinus of the phase, $\cos [\varphi(A)]$, which crosses the horizontal axis at the resonance frequency, a hallmark of harmonic forced oscillators.

For a given shape, the resonance frequency decreases with the aperture size [Fig. 2(c)]. It is higher for solids with small number of faces, such as the tetrahedron. The maximum size of the structure was reached when the aperture size was around $l=2.5 \mathrm{~mm}$, for which capillary forces are not strong enough to prevent water from invading the structures. We found that all results beautifully collapse on the same master curve when plotting the resonance frequency as a function of the size $R_{\text {eq }}$ linked to the gas volume $V_{g}$ [Fig. 2(d)]. The volume is calculated assuming the interfaces are coplanar with the external faces of the solid. We define $R_{\text {eq }}$ as the radius of the sphere with the same volume or $R_{\mathrm{eq}}=\left[V_{g} /(4 \pi / 3)\right]^{1 / 3}$. 
(a)

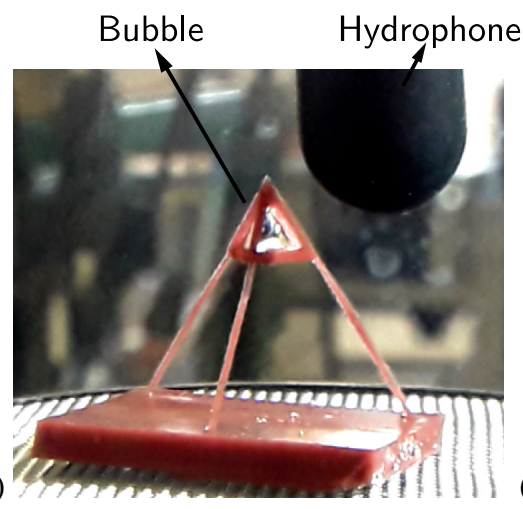

(b)

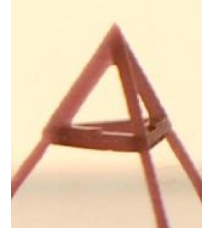

(c)

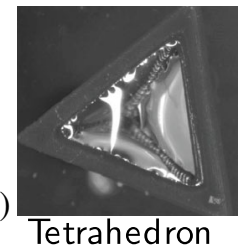

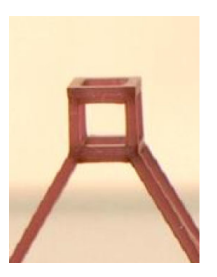

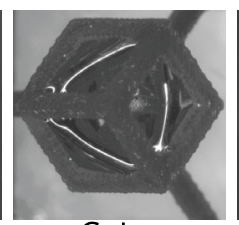

Cube
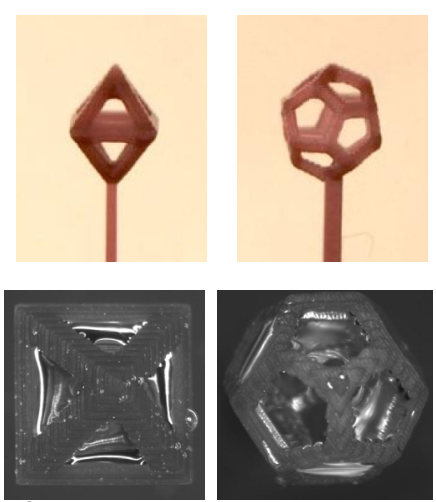

Octahedron

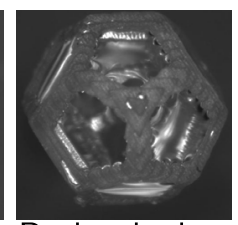

Dodecahedron
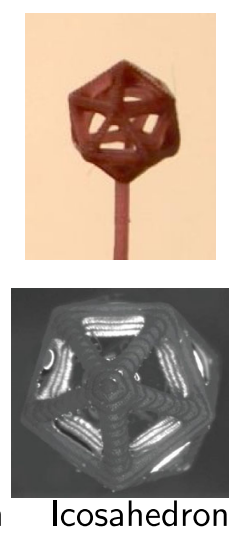

FIG. 1. (a) Immersed tetrahedral bubble and hydrophone. (b) 3D-printed frames for the five Platonic solids. The width of the cube is $3 \mathrm{~mm}$ and gives the scale. (c) After immersion, air-liquid interfaces are pinned at the openings.

Minnert approximation.-Minnaert [1] found that the resonance frequency of a spherical bubble of radius $R_{\mathrm{eq}}$ is given by

$$
f_{\text {Minnaert }}=\frac{1}{2 \pi}\left(\frac{3 \gamma P_{0}}{\rho_{l} R_{\mathrm{eq}}^{2}}\right)^{1 / 2} \simeq \frac{3.24 \mathrm{~m} / \mathrm{s}}{R_{\mathrm{eq}}},
$$

with $\rho_{l}$ the liquid density, $\gamma$ the specific heat ratio, and $P_{0}$ the gas pressure, valid for bubbles larger than a micrometer. This formula is called the Minnaert formula, plotted as a line in Fig. 2(d). The agreement between the measured resonant frequency of Platonic bubbles and that predicted by the Minnaert frequency is stunning even for shapes far from spherical. This formula is thus a very good approximation when using the equivalent radius of our shapes. A closer comparison of the resonance frequency with that of Minnaert is obtained by plotting $f_{\text {res }} / f_{\text {Minnaert }}$ [inset of Fig. 2(d)]. It shows slightly higher frequencies for the tetrahedron, despite measurement noise due to variations of the position of the contact line around each interface. However, all measured resonance frequencies differ from Minnaert frequency by less than $10 \%$.

In order to understand the impact of the shape on the bubble vibration, we conducted numerical 3D simulations of sound propagation, implemented with a finite-difference time-domain resolution $[10,13]$ of elastodynamics equations. (See further details in Supplemental Material [12].) Here the solid frame is neglected (assumed infinitely thin) and the whole Platonic shape is filled with gas. Wideband pressure pulses were sent in the presence and absence of bubbles as in the experiment, to feature the bubble contribution. The emitted pressure wave has isovalues whose shape first match the bubble shape at very close distance, and then rapidly transits to a monopolar spherical emission further away from the bubble (Fig. 3). When looking at the resonance frequency, we observe a significant deviation from Minnaert for the tetrahedron, whose frequency is higher by $8 \%$, further supporting the experimental observation, while it tends to Minnaert for the icosahedron.
We give here a theoretical hint of this behavior, inspired by Ref. [14]. For small pulsations of the gas volume, the gas volume $V_{g}$ oscillates around an equilibrium value $V_{g}^{0}$. The total potential energy writes $U=\frac{1}{2} k\left(V_{g}-V_{g}^{0}\right)^{2}$, with $k$ a stiffness coefficient due to the gas compression (neglecting surface tension), that depends only on the bubble volume. The total kinetic energy of the water moved around the bubble writes $E_{c}=\iiint \frac{1}{2} \rho_{l} v^{2} d V=\frac{1}{2} m \dot{V}_{g}$, with $m$ an inertial coefficient that depends on the exact geometry, being a function of the potential velocity field $v$ produced by the volume pulsation. The bubble natural frequency of the oscillator is given by $f=\sqrt{k / m} / 2 \pi$. Strasberg [14] found an analogy between the coefficient $m$ and the electrostatic capacitance $C$ of the shape: formally $m / \rho_{l}$ is equivalent to the inverse of $C / \epsilon_{0}$, with $\epsilon_{0}$ the vacuum permittivity.

The calculation of the capacitance of polyhedra is a longstanding mathematical challenge [15]. It amounts to solving $C / \epsilon_{0}=-\iint_{\Gamma}(\partial U / \partial n) d S$, the integral over the surface of the normal derivative of $U$, the latter being the solution of Laplace equation $\Delta U=0$ with boundary condition $U=1$ on the surface $\Gamma$. Using numerical solutions [15] for the value of $C$ of polyhedra, we can compute the ratio of the capacitance to the capacitance of the sphere with the same volume $C_{\text {sphere }}\left(R_{\text {eq }}\right)=4 \pi \epsilon_{0} R_{\text {eq }}$, from which we deduce the frequency ratio

$$
\frac{f}{f_{\text {Minnaert }}}=\left(\frac{C}{C_{\text {sphere }}}\right)^{1 / 2}
$$

to be $1.082,1.032,1.027,1.0061,1.0067$, respectively, from tetrahedron to icosahedron, close to sound propagation simulations [Fig. 3(f)]. Note that these ratios are larger than 1, suggesting that the sphere is the shape minimizing the resonance frequency (or the capacitance in the frame of our electrostatic analogy) at a given volume. This hypothesis is supported by exact results for some families of shapes, such as the ellipsoids [16], or in general for a slightly nonspherical shape.

The size of platonic bubbles is limited by water invasion: the biggest one is the icosahedron $(N=20)$ with an 
(a)
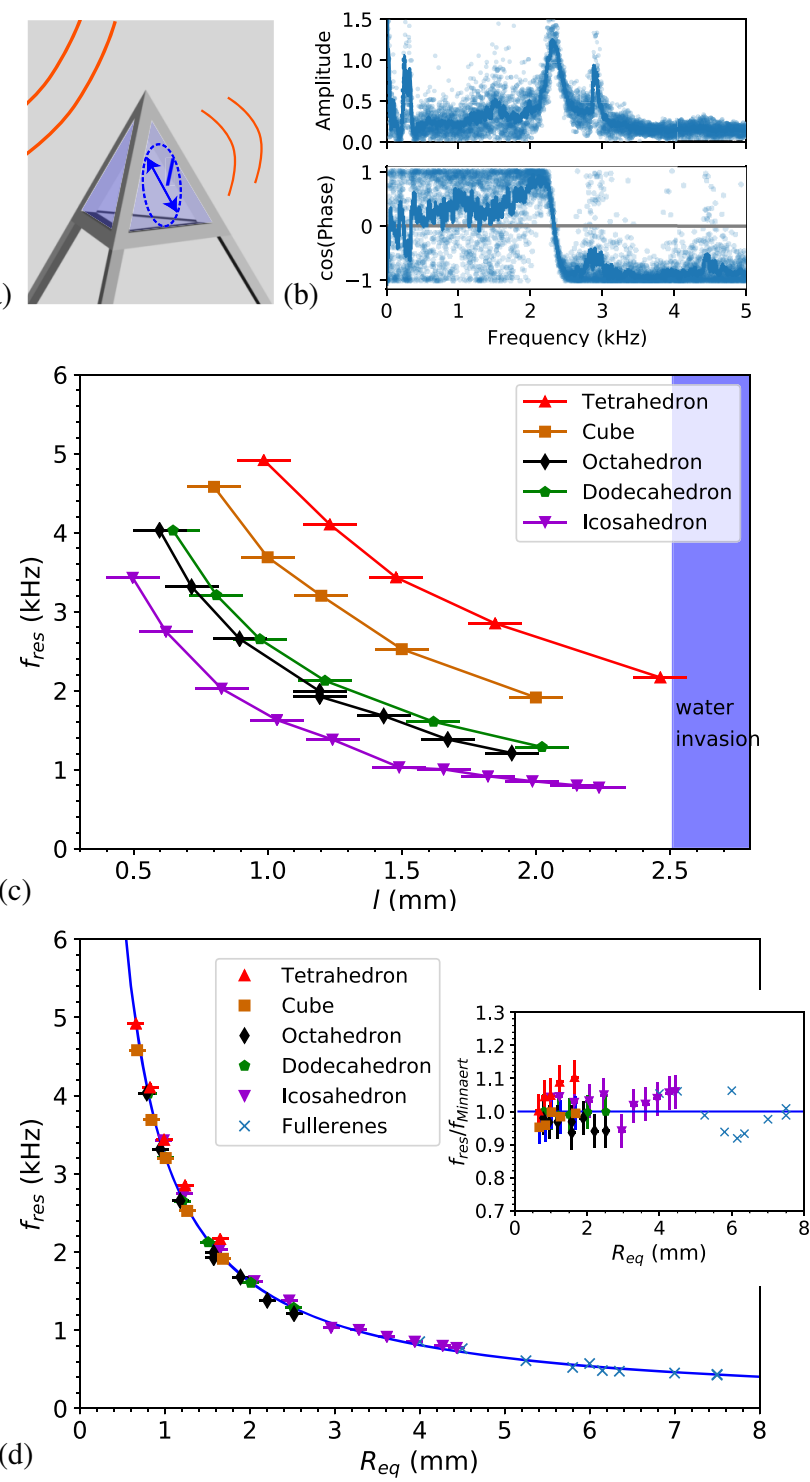

FIG. 2. (a) Vibration of a Platonic bubble, shown here with a tetrahedron. The opening size is measured by the incircle of diameter $l$. (b) Amplitude and cosinus of the phase of the normalized frequency response $A$ for a tetrahedron with $l=2.46 \mathrm{~mm}$. (c) Resonance frequency as a function of $l$ when changing the bubble size. For apertures larger than $2.5 \mathrm{~mm}$ water invaded all the structure. (d) Resonance frequency as function of $R_{e q}$, the radius of the gas volume packed in a sphere. Line: Minnaert model [Eq. (1)]. Cross: fullerenes. Inset: Ratio of the resonance frequency to Minnaert frequency.

equivalent radius $R_{\text {eq }}=4.5 \mathrm{~mm}$, and apertures of diameter $l_{\max }=2.5 \mathrm{~mm}$. Bigger bubbles could be imagined if one could design frames with a larger number of faces, which is not possible with regular polyhedron. This becomes possible if we go beyond Platonic solids.

More faces with the slightly irregular fullerenic solids.We therefore explored slightly irregular shapes considering the shapes of fullerenes, and in particular the ones that are made of hexagons and 12 pentagons positioned

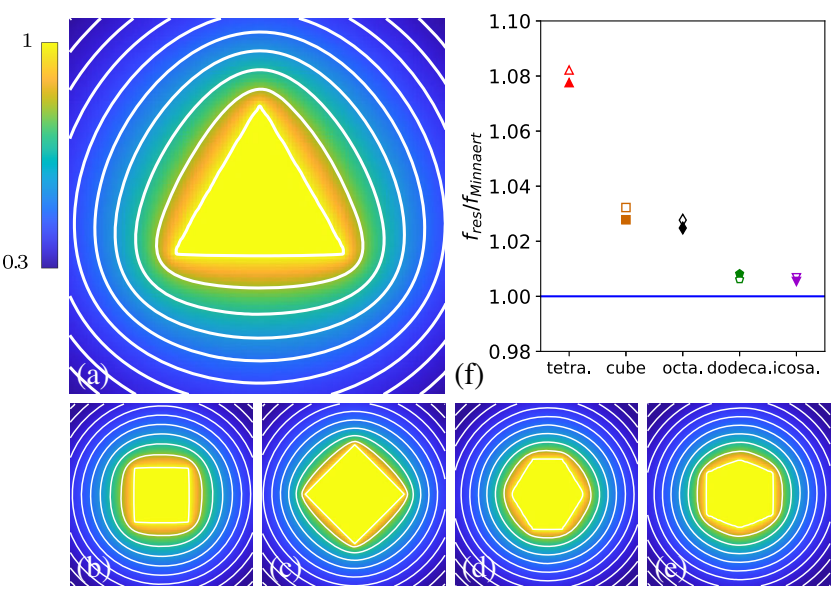

FIG. 3. (a)-(e) Simulation of the pressure field emitted by the vibration of a platonic shape made of gas (cross section in the middle, except for the tetrahedron cut along a face). Each image represents a snapshot of the scattered pressure amplitude at a time of maximum pressure, normalized to the maximum value inside the bubble. Isolines are chosen to be equidistant for a field decaying as $1 / r$. Bubble volume is $27 \mathrm{~mm}^{3}$ made of $0.1 \mathrm{~mm}$ voxels. (f) Frequency as a function of the number of faces, normalized with Minnaert model. Filled symbols: numerical predictions from the finite-difference time-domain simulations. Open symbols: numerical solutions from the electrostatic analogy.

homogeneously on the surface, at the vertices of an inscribed icosahedron. We designed a variety of fullerenes, starting from the smallest one which is the Buckminsterfullerene $\mathrm{C}_{60}$ (a celebrated shape which formed the panels of the Telstar soccer balls used in World Cups 1970 and 1974), then continuing with $\mathrm{C}_{180}$, $\mathrm{C}_{240}, \mathrm{C}_{320}$, and $\mathrm{C}_{540}$, featuring $32,92,122,162$, and 272 faces, respectively; see Figs. 4(a) and 4(b). With fullerenes, we could increase the gas volume up to an equivalent radius $R_{\text {eq }}=7.5 \mathrm{~mm}$ (with $\mathrm{C}_{540}$ ).

The resonant frequency of fullerene bubbles was measured: it is still well approximated by the Minnaert relationship; see Fig. 2(d). Note that such big bubbles feature a high quality factor (around 30) close to the theoretical value for a free spherical bubble of the same size [2]; the presence of a structure does not add much damping.

One may wonder if there is a limit to the size of such fullerene bubbles. We experienced that manufactured fullerenes needed smaller apertures, in comparison to Platonic shapes, to prevent water invasion. The maximum aperture diameter is $l_{\max }=1.45 \mathrm{~mm}$ for $\mathrm{C}_{540}$ instead of $l_{\max }=$ $2.5 \mathrm{~mm}$ for the biggest icosahedric bubble.

This trend can be modeled using the simple following arguments. When the dry frames of radius $R$ are dipped partially in water, interfaces at the bottom experience a larger hydrostatic pressure just before complete immersion [17], of order $2 \rho_{l} g R$. It is thus necessary to have apertures small enough so that capillary Laplace pressure (of order $4 \sigma / l$ for the highest interface curvature) can counteract the 


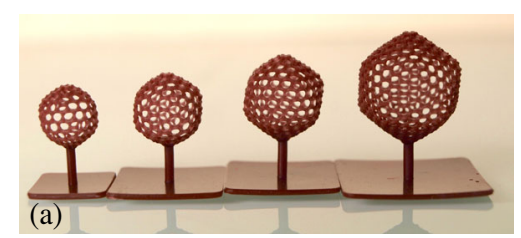

(b)

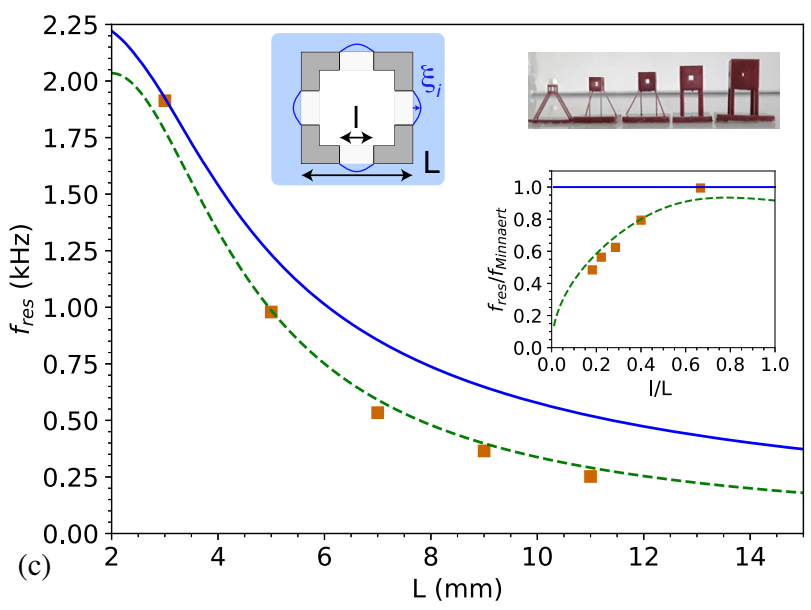

FIG. 4. (a) Fullerenes with a larger number of faces: $\mathrm{C}_{180}, \mathrm{C}_{240}$, $\mathrm{C}_{320}$, and $\mathrm{C}_{540}$. (b) Immersed fullerene $\mathrm{C}_{180}$ and microphone having a diameter of $9 \mathrm{~mm}$, giving the scale. (c) Impact of thicker frames: resonance frequency for increasing face size $L$, using cubes with a constant opening $l=2 \mathrm{~mm}$. Top right photograph: cubes with external sizes $L$ ranging from 3 to $11 \mathrm{~mm}$; the thickness of faces is kept constant with $0.5 \mathrm{~mm}$. Line: Minnaert frequency. Dashes: multifaceted model. Inset: Ratio of the frequency to Minnaert as a function of $l / L$.

hydrostatic pressure, and the aperture should be less than $l \simeq 2 \sigma / \rho_{l} g R$. Numerical data on $l_{\max }$ as a function of $R_{e q}$ closely follow this relationship with a prefactor 0.8 if we chose $\sigma=70 \times 10^{-3} \mathrm{~N} / \mathrm{m}$.

A big polyhedral bubble therefore needs a lot of small apertures. If the aperture area is a fraction $\phi(<1)$ of the Platonic face area, we find the total number of holes to be $N \simeq 4 \pi R^{2} \phi /\left(\pi l^{2} / 4\right)$, assuming all faces to be identical and assuming the shape to be close to a sphere. This number should be higher than $N \sim 4 \phi\left(R / l_{c}\right)^{4}$, with $l_{c}$ the capillary length, to prevent the water invasion. As a conclusion, there is actually no limit on the bubble size, but the number of faces increases dramatically with size.

Multifaceted model for thick edges.-One may also wonder about the acoustic influence of the frames, especially if they become thick. To address this question we have on purpose generated a polyhedron (here a cube) with openings becoming smaller than the face size; see photograph and sketch in Fig. 4(c). We observe that the resonance frequency departs from the Minnaert prediction when the openings become small compared to the faces. A qualitative explanation is that only a smaller proportion of the surface is oscillating compared to the case of a pulsating sphere. This effect can be modeled quantitatively following the approach in Ref. [10] focusing on individual interfaces in the openings, that behave as individual oscillators when they vibrate, coupled through the gas (since they compress the same gas) and through the liquid (since they emit acoustic waves).

According to this approach, each vibrating interface (labeled $i$ ) can be modeled as an individual oscillator whose displacement is $\bar{\xi}_{i}$ (averaged over the surface assumed circular). The gas compression or decompression results in a potential energy $U=\frac{1}{2} k \bar{\xi}_{i}^{2}$ with a stiffness $k=$ $\gamma P_{0}\left(\pi l^{2}\right)^{2} / 4 V_{g}$. When alone, a moving interface entrains the fluid around and involves a kinetic energy $E_{c, i}=$ $\frac{1}{2} m_{i} \bar{\xi}_{i}^{2}$ with an added mass $m_{i}=(4 / 15) \rho_{l} l^{3}$, assuming a circular aperture embedded within infinite plane $[18,19]$. In the lowest mode, the $N$ interfaces vibrate in phase and experience a demultiplied effective stiffness $k_{\text {eff }}=N k$ and an effective mass $m_{\text {eff }}=m+\pi \rho_{l} l^{3} \sum_{j \neq i}\left(l / 64 r_{i j}\right)$, with $r_{i j}$ chosen to be the shortest path in the liquid (for a cube $\left.r_{i j}=L\right)$ [10]. The resonance is computed from $f_{\text {res }}=$ $\left(k_{\text {eff }} / m_{\text {eff }}\right)^{1 / 2} / 2 \pi$ and plotted in Fig. 4(c) as dashes and matches well the experiments. The frequency is lowered compared to Minnaert and even tends to zero when the ratio of the aperture size to face size $l / L$ tends to zero.

Conclusion and perspectives.-The resonance frequency of Platonic and fullerene bubbles is well described by an equation based on the Minnaert formula, in spite of the presence of many frame edges and in spite of significant deviation from sphericity. It is only when edges are thick that we need a specific multifaceted model. A large number of faces is possible, provided the opening is reduced to counteract the additional hydrostatic pressure. Perspectives include the use of this new type of bubbles in acoustic metamaterials, trapping many bubbles at desired positions and dimensions.

P. M. acknowledges financial support from the European Community's Seventh Framework Programme (FP7/20072013) ERC Grant Agreement Bubbleboost No. 614655.

*philippe.marmottant@univ-grenoble-alpes.fr

[1] M. Minnaert, Philos. Mag. J. Sci. 16, 235 (1933).

[2] T. G. Leighton, The Acoustic Bubble (Academic Press, London, 1994).

[3] D. R. Smith, J. B. Pendry, and M. C. K. Wiltshire, Science 305, 788 (2004).

[4] V. Leroy, A. Strybulevych, M. G. Scanlon, and J. H. Page, Eur. Phys. J. E 29, 123 (2009).

[5] A. Bretagne, A. Tourin, and V. Leroy, Appl. Phys. Lett. 99, 221906 (2011).

[6] T. Brunet, A. Merlin, B. Mascaro, K. Zimny, J. Leng, O. Poncelet, C. Aristégui, and O. Mondain-Monval, Nat. Mater. 14, 384 (2014).

[7] V. Leroy, A. Strybulevych, M. Lanoy, F. Lemoult, A. Tourin, and J. H. Page, Phys. Rev. B 91, 020301(R) (2015).

[8] P. S. Epstein and M. S. Plesset, J. Chem. Phys. 18, 1505 (1950). 
[9] C. E. Brennen, Cavitation and Bubble Dynamics (Oxford University Press, Oxford, 1995).

[10] M. Harazi, M. Rupin, O. Stephan, E. Bossy, and P. Marmottant, Phys. Rev. Lett. 123, 254501 (2019).

[11] T. Combriat, P. Rouby-Poizat, A. A. Doinikov, O. Stephan, and P. Marmottant, Soft Matter 16, 2829 (2020).

[12] See Supplemental Material at http://link.aps.org/ supplemental/10.1103/PhysRevLett.126.054502 for more details on frame design and fabrication, acoustic measurements and numerical simulations.

[13] E. Bossy, SimSonic, A FDTD simulation freeware, http:// www.simsonic.fr.
[14] M. Strasberg, J. Acoust. Soc. Am. 25, 536 (1953).

[15] C. Brown, Comput. Math. Appl. 20, 43 (1990).

[16] Electrodynamics of Continuous Media, edited by L. Landau and E. Lifshitz, Course of Theoretical Physics, 2nd ed., Vol. 8 (Pergamon, Amsterdam, 1984).

[17] Z. Cai, S. Zhao, Z. Huang, Z. Li, M. Su, Z. Zhang, Z. Zhao, X. Hu, Y.-S. Wang, and Y. Song, Adv. Funct. Mater. 29, 1906984 (2019).

[18] D. L. Miller and W. L. Nyborg, J. Acoust. Soc. Am. 73, 1537 (1983).

[19] H. Gelderblom, A. G. Zijlstra, L. van Wijngaarden, and A. Prosperetti, Phys. Fluids 24, 122101 (2012). 